Onkologe $2011 \cdot 17: 282-283$

DOI 10.1007/s00761-011-2016-x

Online publiziert: 25. März 2011

(c) Springer-Verlag 2011

\author{
A.-L. Grosu' $\cdot$ K. Höffken ${ }^{2}$ \\ ${ }^{1}$ Klinik für Strahlenheilkunde, Radiologische Klinik, Universitätsklinikum Freiburg \\ 2 Universitätsklinikum Jena
}

\title{
Radikalität und Funktionalität - zwei Seiten einer Medaille
}

In keiner Körperregion ist die Relation zwischen Morphe und Funktion so prägnant wie im ZNS-Bereich. Das Ziel der modernen Neurochirurgie ist, nicht nur den Tumor vollständig zu entfernen, sondern v. a. die Funktion der anatomischen Region maximal zu erhalten. Nach Teilresektion kann in vielen Fällen eine ergänzende lokale, fokussierte Strahlentherapie eine hohe lokale Tumorkontrolle unter Schonung der funktionellen Areale erreichen. Die Radioonkologie hat die positiven, aber auch die negativen Folgen der Bestrahlung in zahlreichen Studien evaluiert und die Konsequenzen daraus gezogen: die Radikalität einer Strahlenbehandlung sollte im klinischen Kontext definiert werden. Die neuen systemischen Therapien, primär oder in Ergänzung zu den anderen Behandlungsmethoden, versuchen tumorspezifische Rezeptoren $\mathrm{zu}$ identifizieren und dadurch unerwünschte Nebenwirkungen zu reduzieren.

Alle diese Konzepte können sich nur im Dialog unter den verschiedenen Disziplinen entwickeln und etablieren. Kooperation und interdisziplinäres Denken sind essenzielle Charakteristika der modernen Onkologie. Alle therapeutischen Strategien müssen in Tumorkonferenzen in Konsens zwischen den verschiedenen diagnostischen und therapeutischen Disziplinen definiert werden. Das gilt sowohl für die individuellen Behandlungskonzepte als auch für die großen multizentrischen randomisierten Phase-III-Studien.

Mit der vorliegenden Ausgabe von Der Onkologe wird bereits zum 2. Mal in diesem Jahr das wichtige Thema „Tumoren des Zentralen Nervensystems (ZNS)“ aufgegriffen - mit dem Ziel, neue, innovative Aspekte in der Diagnostik und Behandlung von ZNS-Erkrankungen zu präsentieren. Von Hirnmetastasen (Grosu et al.) und Meningiosis carcinomatosa (Mügge et al.) bis zu ZNS-Lymphomen (Illerhaus et al.), PET-Diagnostik (Weber et al.) oder intraoperative Funktionslokalisierung (Vajkoczy et al.) werden die vorliegenden Beiträge die Leser über die neuen Entwicklungen in der Behandlung von Hirnneoplasien informieren.

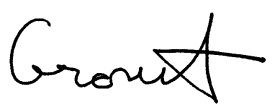

A.-L. Grosu

Für die Herausgeber des Schwerpunktheftes

Ce. Horron

K. Höffken

Für die Herausgeber

\section{Korrespondenzadresse}

Prof. Dr. A.-L. Grosu

Klinik für Strahlenheilkunde, Radiologische Klinik, Universitätsklinikum Freiburg Robert-Koch-Straße 3, 79106 Freiburg anca.grosu@uniklinik-freiburg.de 
Hier steht eine Anzeige.

黛 Springer 